\title{
Dissipative particle dynamics: dissipative forces from atomistic simulation
}

\author{
Vlad P. Sokhan and Ilian T. Todorov
}

\section{Published version information}

Citation: VP Sokhan and IT Todorov. Dissipative particle dynamics: dissipative forces from atomistic simulation. Mol Simul 47, no. 2-3 (2019): 248-256

DOI: $\underline{10.1080 / 08927022.2019 .1578353}$

This is an Accepted Manuscript of an article published by Taylor \& Francis in Molecular Simulation on $18^{\text {th }}$ February 2019, available online:

http://www.tandfonline.com/10.1080/08927022.2019.1578353.

This version is made available in accordance with publisher policies. Please cite only the published version using the reference above. This is the citation assigned by the publisher at the time of issuing the AAM/APV. Please check the publisher's website for any updates. 
To appear in Molecular Simulation

Vol. 00, No. 00, Month 20XX, 1-13

\title{
Dissipative particle dynamics: Dissipative forces from atomistic simulation
}

\author{
Vlad P. Sokhan and Ilian T. Todorov \\ STFC Daresbury Laboratory, Sci-Tech Daresbury, Keckwick Lane, Daresbury, Cheshire WA4 4AD \\ (Received 00 Month 20XX; final version received 00 Month 20XX)
}

\begin{abstract}
We present a novel approach of mapping dissipative particle dynamics (DPD) into classical molecular dynamics. By introducing the invariant volume element representing the swarm of atoms we show that the interactions between the emerging Brownian quasiparticles arise naturally from its geometric definition and include both conservative repulsion and dissipative drag forces. The quasiparticles, which are composed of atomistic host solvent rather than being simply immersed in it, provide a link between the atomistic and DPD levels and a practical route to extract the DPD parameters as direct statistical averages over the atomistic host system. The method thus provides the molecular foundations for the mesoscopic DPD. It is illustrated on the example of simple monatomic supercritical fluid demonstrating good agreement in thermodynamic and transport properties calculated for the atomistic system and DPD using the obtained parameters.
\end{abstract}

Keywords: Dissipative particle dynamics; molecular dynamics; coarse-graining; parameters estimation; DL_POLY

\section{Introduction}

As is well known, the principal workhorse of computer modelling of condensed matter - molecular dynamics - works best when all degrees of freedom are of equal denomination. When the problem includes both fast (local) and slow (collective) degrees of freedom and they are coupled, as is often the case in condensed matter problems [1], the efficiency of the statistical sampling dramatically reduces. Finding efficient coarse-grained (CG) solutions, i.e., based on reduced and more homogeneous sets of variables, is a challenging problem that has attracted much attention in recent years [2]. In general, CG leads to the generalised Brownian motion of the remaining variables [3], which in practical terms implies a realisation of a stochastic process governed by a generalised Langevin equation (GLE) [4]. Direct application of the projection operator approach of Zwanzig [5] and Mori [6] to derive the memory kernel of the GLE is a highly nontrivial problem [7] which can be alleviated by accepting the Markovian approximation (but see [8]).

When the dynamics is not of primary importance and only the thermodynamic properties are involved, the solution is provided by a mapping of free energies defined in full and reduced phase spaces [9, 10]. Examples include polymer solutions [11-15], colloidal systems [9] and biological materials [2, 16-18], commonly known as soft matter. There, a functional group of chemically connected atoms is typically replaced by a single bead, and several CG force fields, including MARTINI [19], UNRES [20, 21] and SIRAH [22], systematically explore this approach. Many methods exist for deriving CG forces from the atomistic level, including the force-matching method [23], reverse Monte Carlo [24, 25] and relative entropy minimisation [26]. These CG methods are able to provide accurate structure for polymers and biomolecular systems and have demonstrated significant speed-up due both to the smoothened energy landscape in the reduced configuration space as well as the increased timestep. With additional tuning, they are also capable of correctly 
reproducing the fluid structure.

In contrast, deriving methods for an accurate and faithful CG dynamics appears to be a more complex problem for which no general solution exists as yet. The essence of the problem lies at the heart of CG itself. Simple elimination of some of the degrees of freedom (DOF) in the system takes away the associated channels of energy relaxation which results in accelerated transport properties. As a result, the CG dynamics is often an order or two faster than the corresponding atomistic one [27-29]. While in some cases this might be an advantage, for example by allowing fast determination of structure or thermodynamic properties, this precludes its application to fluid dynamics problems. In addition, as has been shown early on by Dünweg [30], modification of dynamics due to thermostats that violated Galilean invariance generally results in artificial hydrodynamic screening.

To accurately capture the hydrodynamic effects at the coarse-grained level, several methods have been proposed [31], of which Dissipative Particle Dynamics (DPD) [32] is particularly suitable for the study of dynamic and transport properties of the complex soft matter at the mesoscale. In its recent form [33, 34] the method extends Brownian dynamics [3] to incorporate hydrodynamic effects through a Galilean invariant thermostat [35], which consequently stipulates the isothermal conditions. The basis for this approach stems from the fact that repulsive forces alone can describe the structure of liquids, as has been profusely demonstrated in the WCA theory [36], and combined with a local short-ranged pairwise Langevin thermostat this makes DPD an attractive, efficient tool for multiscale interrogation of soft matter. The advantages and limitations of the method have been discussed in a recent review [37].

Predictive power of the method depends critically on our ability to relate the model parameters to a specific chemistry, to derive them from first principles. Interaction between DPD particles includes both conservative and Langevin forces and while several methods were proposed for the former [38-40], the solution to the latter problem still eluded us [41]. The problem is particularly acute for coarse-graining the non-bonded DOF in simple fluids, e.g., swarms of atoms or small molecules [41]. Existing methods, depending on the thermostat used, often result in diffusivities that are too high (viscosities that are too low) [42]. It is also khown that the Markovian approximation employed in DPD equations of motion is unsound in the case of a linear force of the DPD ansatz [8].

Here, we present a new general method of deriving the DPD model parameters, including dissipative forces, from atomistic simulation. In the method, we first define a Brownian quasiparticle (BQ) in the host atomic fluid: a CG object of constant volume, which both consists of fluid atoms and is immersed in the host fluid. The BQ is therefore an open system of fluctuating mass. Its motion, and more precisely its kinematics, is determined by the gross motion of the constituent atoms and from the analysis of its motion the parameters of the corresponding Brownian motion can be estimated. Translation of these parameters into the DPD ansatz fixes both the analytic shape of DPD forces and their values.

\section{Dissipative Particle Dynamics and the Brownian quasiparticles}

Dissipative particle dynamics is a mesoscopic particle-based method aimed primarily at hydrodynamic and rheological modelling of the fluid phase. In it, the atomistic nature of fluids is seen through the blurry looking glass of coarse-graining. As a result, despite the widespread usage of the method there is no precise definition of its fundamental constituent, the DPD particle itself. While in coarse-grained methods for bonded species a 'group of atoms' [37] or molecular moiety is often used to define a coarse-grained bead, the situation is far less clear-cut for non-bonded atoms [41, 43], where a 'lump of fluid' [43, 44] or a 'fluid element' [29], apparently making implicit reference to continuum hydrodynamics, is termed as a DPD particle.

In a sense, the DPD particle is isomorphic to the 'illuminated volume' [1] of light-scattering or 
inelastic coherent neutron scattering experiments, both sensitive to the local density fluctuations in the liquid. The existence of phonons in liquid is well established [45], and although their lifetime is short, we want to exploit this analogy when defining the Brownian quasiparticle. Since the DPD is not a space-filling model, the density of DPD particles is a free parameter and we will utilise this freedom in defining a suitable density range.

In this section our starting point is an ensemble of atoms in a certain thermodynamic state which we 'illuminate' with a mapping ensemble of Brownian quasiparticles. We describe then how the interactions between Brownian quasiparticles and with the surrounding reservoir, mediated by exchanging host atoms, emerge from the definition the BQ. Molecular dynamics of the host subsystem drives the motion at the coarse-grained level and from the analysis of this motion we construct the conservative and dissipative drag forces which are consistent with the target DPD model.

\subsection{Mapping into atomistic system}

Despite the fact that the Brownian quasiparticle shares much in common with the usual Brownian particle, there is one important difference - whereas a Brownian particle is just immersed in fluid, BQ is both immersed and consists of fluid. As a consequence of this, the interactions between the $\mathrm{BQ}$ beads bear the birthmark of the primordial molecular level. Implicitly, they are distilled from a more fundamental atomistic representation through a coarse-graining transformation, and in order to distinguish them we shall call from now on fundamental level objects atoms (irrespective of whether they are atoms or molecules), reserving the term beads (or DPD particles) to the coarsegrained level. For simplicity, we shall also assume that particles are spheres, although they could take any convex shape as long as their volume remains invariant.

In the original papers $[32,46]$ very little details were given about the relation between the DPD particles and atoms and they were assumed to represent some mesoscopic degrees of freedom, or 'lumps' of fluid [44]. In order to map DPD particles into the atomistic system we propose the following definition: a Brownian quasiparticle collectively represents all atoms contained at a particular moment of time in an invariant volume element of the fluid and its kinematics is defined by the collective motion of the atoms contained in the volume element. This definition is based on the assumption that the interactions between 'lumps of fluid' are short-ranged; this has been postulated in the DPD model and although in the BQ picture indirect interactions extend through the host fluid leading to a 'hydrodynamic tail', direct intervention ceases at some separation $a$ taken as the BQ size. The definition also implies that the mass of the quasiparticle and its 'content' fluctuates in time according to the grand canonical conditions (open system), and that its velocity at a particular instant of time is given by a velocity centroid of all constituent atoms. The last statement, as will be shown later, introduces a bias in the particle number distribution and therefore in the chemical potential of the Brownian quasiparticle.

Using a loose analogy with gauge bosons in particle physics, we define the interactions between quasiparticles in terms of the exchange of 'virtual particles', which in this case are atoms, only 'virtually' present at the coarse-grained level. There are two types of quasiparticle interactions depending on whether the atom leaving the volume element goes into the 'reservoir' or exchanges with another volume element. The former describes the act of interaction with the 'environment', or 'heat bath', and contributes to Langevin's friction and noise, and the latter is the classical analogue of 'exchange repulsion', which could be understood on the example of two boats on the lake exchanging watermelons by throwing. We note in passing that similar ideas were used to illustrate the origin of the shear viscosity (e.g., the 'train exchange' example on p. 303 of Tabor's book on gases, liquids and solids[47]). However, since our target, the DPD ansatz, does not provision for shear forces, the dissipative forces of DPD are related to bulk viscosity only. Also, momentum conservation requires that each atom could be either a member of the heat bath or belong to one 
(a)

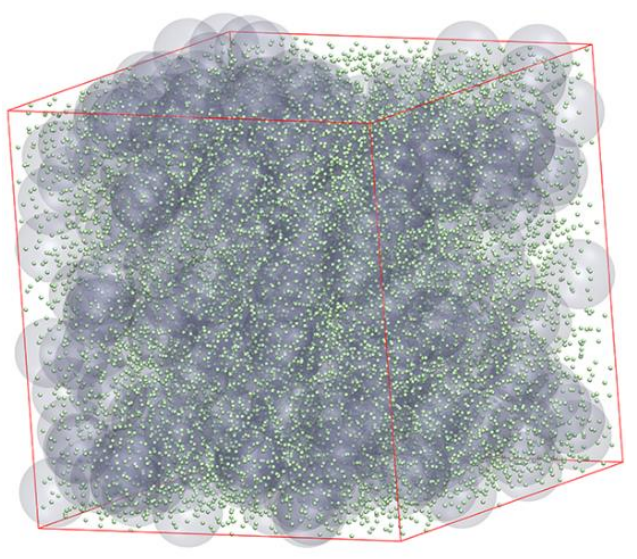

(b)

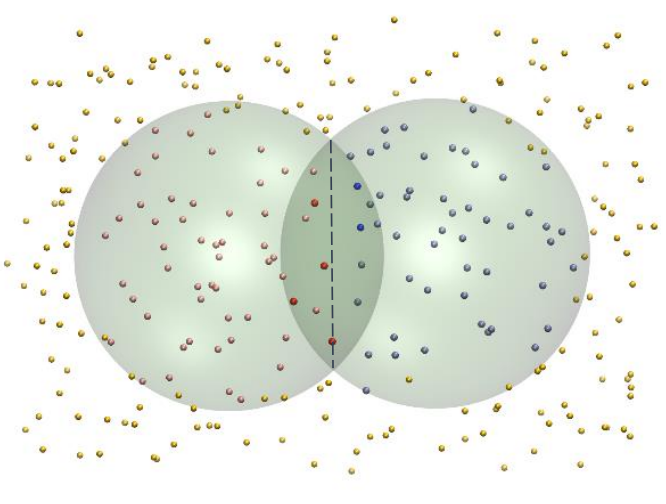

Figure 1. (Colour online) (a) Sketch of the model. Large transparent spheres represent the Brownian quasiparticles, small beads - LJ particles, and the system is periodically replicated in 3D. (b) Illustration of the two overlapping BQs. Dashed line denotes the position of the dividing plane separating the volumes of two quasiparticles. Atoms belonging to each quasiparticle are distinguished by their colour and in the overlap volume atoms belonging to the left BQ are red and those belonging to the right $\mathrm{BQ}$, blue.

BQ only and cannot be in two particles simultaneously. This condition is sufficient to devise the interactions between BQ beads. When BQ spheres overlap, the atoms are reassigned according to the Voronoi tessellation [39], i.e., each atom belongs to the nearest sphere.

The above method exposes one of the intrinsic shortcomings of the DPD model, in which the interacting Brownian particles suspended in a solvent are modelled by an ensemble of interacting Brownian particles in vacuo. In the low-density limit, a DPD particle, stripped of its heat bath interactions, behaves like an ideal classical particle, travelling ballistically, whereas a BQ of the described model will retain the interactions with the environment, leading to diffusive Brownian motion. As a result, an ideal dissipative gas (i.e., DPD particles without conservative forces) presents a completely different limiting case from the gas of ideal Brownian quasiparticles, whose interactions are guided by the long-range and nonlocal hydrodynamic effects in the underlying atomistic system.

\subsection{Estimating DPD forces in molecular dynamics}

Consider an ensemble of $N$ atoms in volume $V$ prepared in initial state by coupling to a thermostat at temperature $T$. After initial equibration the system is isolated and evolved further according to constant energy molecular dynamics. In the production section, we disperse across the system $M$ BQ 'watchers' (we are interested in $M<<N)$ of mass $m_{i},(i \in[1, M]$ ), and size $a$, which are large in comparison with the corresponding atomic quantities as sketched in Figure 1(a). Motion of atoms is unaffected by the presence of coarse-grained watchers while the motion of the latter obeys the Langevin equations of motion [4],

$$
\begin{gathered}
\dot{\mathbf{r}}_{i}(t)=\mathbf{p}_{i}(t) / m_{i}, \\
\dot{\mathbf{p}}_{i}(t)=\mathbf{F}_{i}\left(\mathbf{r}_{1}, \ldots, \mathbf{r}_{M}, \dot{\mathbf{r}}_{1}, \ldots, \dot{\mathbf{r}}_{M}\right),
\end{gathered}
$$


where a dot denotes the time derivative and the force on the $i$-th particle, $\mathbf{F}_{i}$, depends on positions and velocities of all other particles in the system. In the pairwise approximation of DPD it is

$$
\mathbf{F}_{i}\left(\mathbf{r}_{1}, \ldots, \mathbf{r}_{M}, \dot{\mathbf{r}}_{1}, \ldots, \dot{\mathbf{r}}_{M}\right)=\sum_{j \neq i} \mathbf{f}_{i j}\left(r_{i j}\right)
$$

where the pair force between the particles $i$ and $j, \mathbf{f}_{i j}$, acting along the interparticle vector $\mathbf{r}_{i j} \equiv$ $\mathbf{r}_{j}-\mathbf{r}_{i}$, consists of three collinear components: conservative, $\mathbf{f}_{i j}^{(\mathrm{c})}$, dissipative, $\mathbf{f}_{i j}^{(\mathrm{d})}$, and stochastic, $\mathbf{f}_{i j}^{(\mathrm{s})}$,

$$
\mathbf{f}_{i j}\left(r_{i j}\right)=\mathbf{f}_{i j}^{(\mathrm{c})}\left(r_{i j}\right)+\mathbf{f}_{i j}^{(\mathrm{d})}\left(r_{i j}\right)+\mathbf{f}_{i j}^{(\mathrm{s})}\left(r_{i j}\right),
$$

where the last two terms, collectively defining the Langevin forces in the DPD ansatz, form a DPD thermostat [35]. Individual force terms are written as

$$
\begin{gathered}
\mathbf{f}_{i j}^{(\mathrm{c})}\left(r_{i j}\right)=\alpha\left(r_{i j}\right) \mathbf{e}_{i j}, \\
\mathbf{f}_{i j}^{(\mathrm{d})}\left(r_{i j}\right)=-\gamma\left(r_{i j}\right) u_{i j} \mathbf{e}_{i j}, \\
\mathbf{f}_{i j}^{(\mathrm{s})}\left(r_{i j}\right)=\sqrt{2 k_{\mathrm{B}} T \gamma\left(r_{i j}\right) / \Delta t} \theta_{i j} \mathbf{e}_{i j} .
\end{gathered}
$$

Here, we slightly modified the standard notation [33, 34] to accommodate more general functional forms, $\mathbf{e}_{i j}$ is a unit vector in the direction of the interparticle vector $\mathbf{r}_{i j}$, i.e., $\mathbf{e}_{i j}=\mathbf{r}_{i j} / r_{i j}$, and $r_{i j} \equiv\left|\mathbf{r}_{i j}\right|$ is the inteparticle separation. Note that the dissipative force (4b) is linear in the relative velocity of two particles along the line conneting them, $u_{i j} \equiv \mathbf{u}_{i j} \mathbf{e}_{i j}$. In the stochastic term (4c) $\theta_{i j}$ is a random variable, independent for each pair of particles, with zero mean and unit variance, i.e., $\left\langle\theta_{i j}(t) \theta_{k l}\left(t^{\prime}\right)\right\rangle=\left(\delta_{i j} \delta_{i j}+\delta_{i j} \delta_{i j} \delta\left(t^{\prime}-t\right)\right)$, where $\delta(t)$ is a Dirac delta function, and a tensor $\delta_{n m}$ is the Kronecker delta.

The model thus includes two functions, $\alpha\left(r_{i j}\right)$ and $\gamma\left(r_{i j}\right)$, whose functional form, together with the required parameters, we want to derive from the underlying atomistic simulation rather than postulate it by accepting, e.g., the standard DPD ansatz. They define conservative and dissipative forces, respectively, of the DPD model.

Since in both atomic and coarse-grained descriptions we are concerned with pairwise forces only, conservative forces could be found in a simplified way assuming the conjecture that manybody effects arise in the same way at both levels and we need to fix the force in the low-density limit only. In that case the conservative part of the interaction potential $U(r)$ between the Brownian quasiparticles is related to their radial distribution function $g(r)$ via Boltzmann inversion,

$$
U(r)=-k_{\mathrm{B}} T \ln g(r),
$$

where $k_{\mathrm{B}}$ is Boltzmann's constant and $T$ is the system temperature. This is known to define uniquely the corresponding forces [48]. In practical terms, we first fit the calculated $g(r)$ to an analytic expression and then use its derivative to estimate the force. This route requires accurate determination of the structure since the accuracy of forces could be severily compromised due to the second step.

For the dissipative forces a suitable procedure can be established by noticing that the dissipative term is the only odd term with respect to time reversal. Therefore, by multiplying eq. (3) through 
Table 1. Simulation parameters and properties of Brownian quasiparticles.

\begin{tabular}{ccccccc}
\hline$a / \sigma$ & $M$ & $N_{m}^{\text {id }}$ & $N_{m}(1)$ & $N_{m}(0)$ & $T_{\mathrm{B}}^{*}$ & $D_{\mathrm{B}}^{*}$ \\
\hline 2 & 3375 & 2.0944 & 2.098 & 2.5482 & 1.173 & 0.075 \\
3 & 999 & 7.0686 & 7.075 & 7.4692 & 1.063 & 0.043 \\
4 & 422 & 16.755 & 16.77 & 17.139 & 1.021 & 0.020 \\
6 & 125 & 56.549 & 46.46 & 56.827 & 0.999 & 0.089 \\
8 & 54 & 134.04 & 110.04 & 134.41 & 0.992 & 0.005 \\
\hline
\end{tabular}

by $\mathbf{u}_{i j}$ and taking the canonical average, one obtains

$$
\left\langle\mathbf{f}_{i j}\left(r_{i j}\right) \mathbf{u}_{i j}\right\rangle=\left\langle\mathbf{f}_{i j}^{(\mathrm{d})}\left(r_{i j}\right) \mathbf{u}_{i j}\right\rangle=-\gamma\left(r_{i j}\right)\left\langle\mathbf{u}_{i j}^{2}\right\rangle
$$

where we took into account that both $\left\langle\mathbf{u}_{i j}\right\rangle=0$ and $\left\langle\theta_{i j} \mathbf{u}_{i j}\right\rangle=0$. Thus, the dissipative force can be estimated in atomistically driven coarse-grained simulation by taking the direct ensemble average,

$$
\gamma\left(r_{i j}\right)=-\frac{\left\langle\mathbf{f}_{i j}\left(r_{i j}\right) \mathbf{u}_{i j}\right\rangle}{\left\langle\mathbf{u}_{i j}^{2}\right\rangle} .
$$

The total force between two beads, $\mathbf{f}_{i j}$, can be directly estimated from its definition,

$$
\mathbf{f}_{i j}\left(r_{i j}\right)=\sum_{\substack{n \in \Omega_{i}, m \in \Omega_{j}}} \mathbf{f}_{n m}\left(r_{n m}\right),
$$

i.e., as a sum over atomic pairs belonging to BQ beads $i$ and $j$, respectively. Similarly, velocity of bead $i$ is calculated as a centroid velocity,

$$
\mathbf{u}_{i}(t)=\sum_{n \in \Omega_{i}} \mathbf{v}_{n}(t)
$$

\section{Simulation results and discussion}

In order to verify and validate the method, as a test case we used, as a host, a simple supercritical Lennard-Jones (LJ) fluid, for which both thermodynamic and transport properties are well known. In standard notation, $\sigma$ and $\epsilon$ define the lendth and energy scales at this level. Single host dynamics drives the coarse-grained motion at various levels defined by the coarse-graining factor $N_{m}$, i.e., an average number of atoms in a BQ bead. This provides a direct way to calculate the distribution functions and from them the potential parameters for the mapping beads.

\subsection{Atomistic system}

We considered a single supercritical state of the LJ fluid at reduced temperature $T^{*}=1.8$ and reduced density $\rho *=0.5$. The system of 13500 particles was placed in a $30 \times 30 \times 30$ cube (in LJ units, see Appendix A), periodically replicated in 3D, and a set of microcanonical MD calculations was performed using the velocity Verlet [3] scheme for reduced times $t^{*}=4.5 \cdot 10^{6}$ using a time step $\Delta t^{*}=3.73 \cdot 10^{-3}$. The interactions were truncated at $2.5 \sigma$, which, together with the small time step used, provided good energy conservation with standard relative deviation in energy per particle of $10^{-6}$. The system was initially equilibrated for $t^{*}=10^{4}$ using the Nosé-Hoover thermostat [3] with 

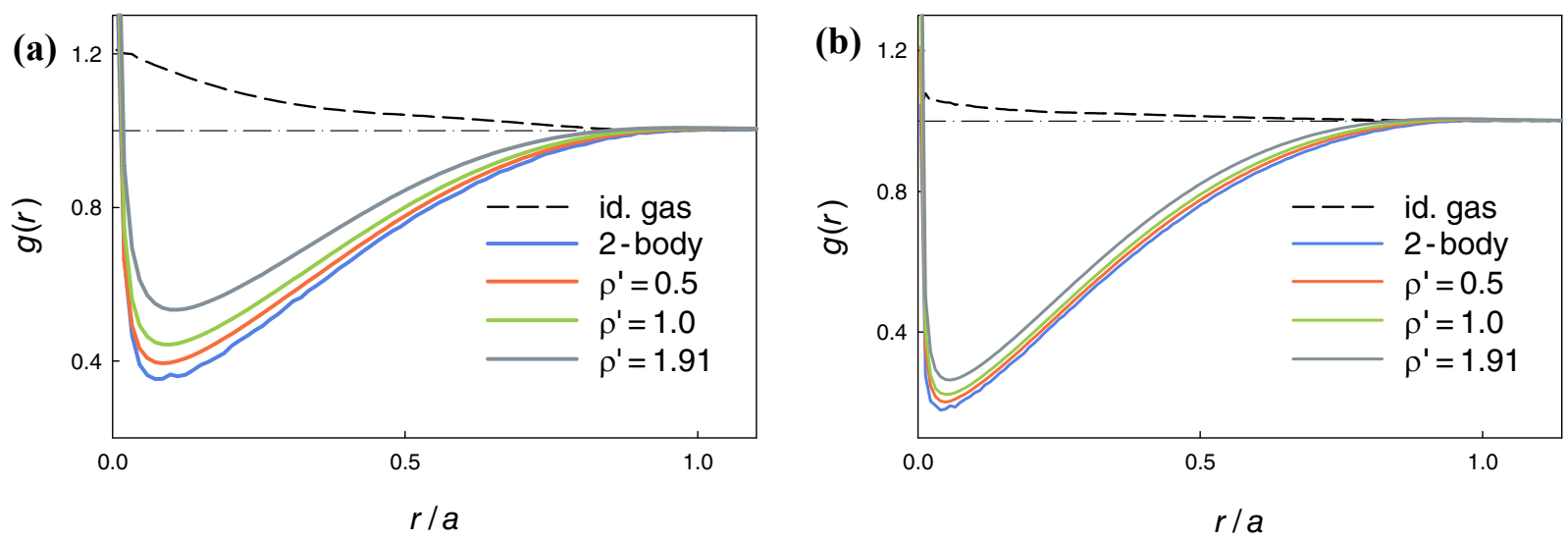

Figure 2. (Colour online) Radial distribution functions (RDFs) for several values of DPD density for: (a) BQ size $a=2 \sigma$, and (b) $a=3 \sigma$. Id. gas denotes the results for BQs that do not interact directly (see text) and 2-body is calculated in the low-density limit where rigorously only two-body forces are present.

relaxation constant of $\tau_{\mathrm{NH}}^{*}=2.3$. In subsequent production runs we monitored the temperature but despite the long MD integration times we did not notice any observable temperature drift.

All calculations were performed using a modified version of DL_POLY [49]. System pressure, calculated using the virial route and including standard long-range corrections for homogeneous systems [3], was $p^{*}=0.7993$.

\subsection{Brownian quasiparticles}

We calculated the structure of the BQ subsystem for a range of quasiparticle sizes from $a=2 \sigma$ to $a=8 \sigma$; they are listed in the first column of Table 1 . In order to determine the appropriate density range for the matching $\mathrm{BQ}$ system we recorded coarse-grained dynamics for densities (in DPD units, see Appendix A) $\rho^{\prime}=0.5,1.0$, and 1.908 (the last is a space-filling equivalent with a volume fraction $\zeta \equiv \pi \rho^{\prime} / 6=1$ [32]). The number of BQ beads used in simulation depends both on the coarse-graining ratio and the density of BQ. The second column of Table 1 gives these values for $\rho^{\prime}=1.0$, and for other densities the numbers scale accordingly. From the definition of the interbead interactions it is clear that the coarse-graining ratio depends on density, and columns 3 to 5 of the Table give values for the 'ideal' coarse-graining ratio, $N_{m}^{\mathrm{id}}=\rho^{*} a^{* 3}$, a purely geometric quantity which is equivalent to averaging over beads randomly distributed through the system, as well as the values for density $\rho^{*}=1, N_{m}(1)$, and for an ensemble of diffusing quasiparticles that do not 'see' each other and interact only with the host system, $N_{m}(0)$. Note that in the last case quasiparticles effectively interact via hydrodynamic fluctuation forces [50] resulting in short-range spatial correlations, as evidenced by their radial distribution funtions (RDF) shown by dashed lines in Figure 2. As a result, the values for $N_{m}(0)$ are always higher than the corresponding ideal values although the effect decreases for larger values of $a$.

To verify that the system-size effects do not introduce any bias in calculated properties at the highest density (volume fraction 1 ) for each value of $a$ we repeated the calculations using a smaller host system containing 4000 atoms in a cubic cell of dimensions $20 \times 20 \times 20$ with proportionally reduced number of quasiparticles. We found no observable differences in the BQ structure.

Figure 2 presents the results obtained for the BQ radial distribution function calculated for several densities for two smaller BQ sizes: (a), $a=2 \sigma$ and (b), $a=3 \sigma$. RDF density dependence, which is a manifestation of manybody effects, is weaker for larger quasiparticles. When the coarsegrained subsystem consists of two particles only, their radial distribution function, as follows from the Henderson's theorem [48], is uniquely defined from their interaction. In this case the potential 


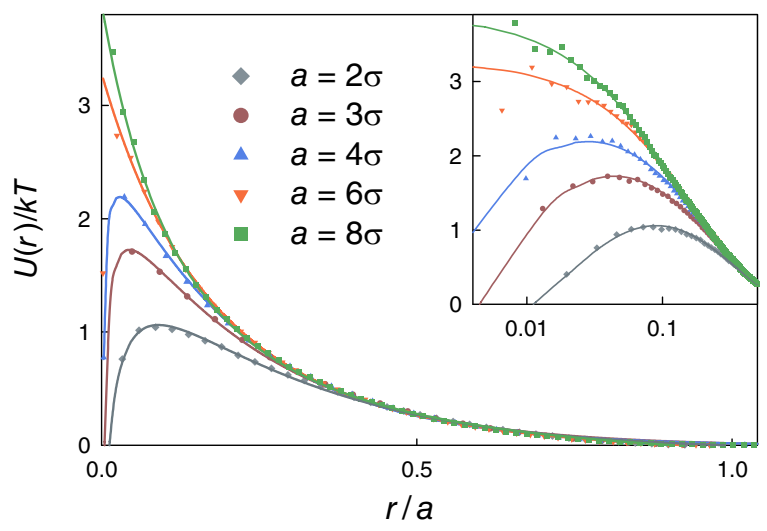

Figure 3. (Colour online) Potential of mean force for DPD particles of several dimensions. Symbols - simulation results, lines - truncated exponential fit using Equation (B1). The inset shows initial part of the distributions at low BQ separations, note the $\log$ scale for the separation.
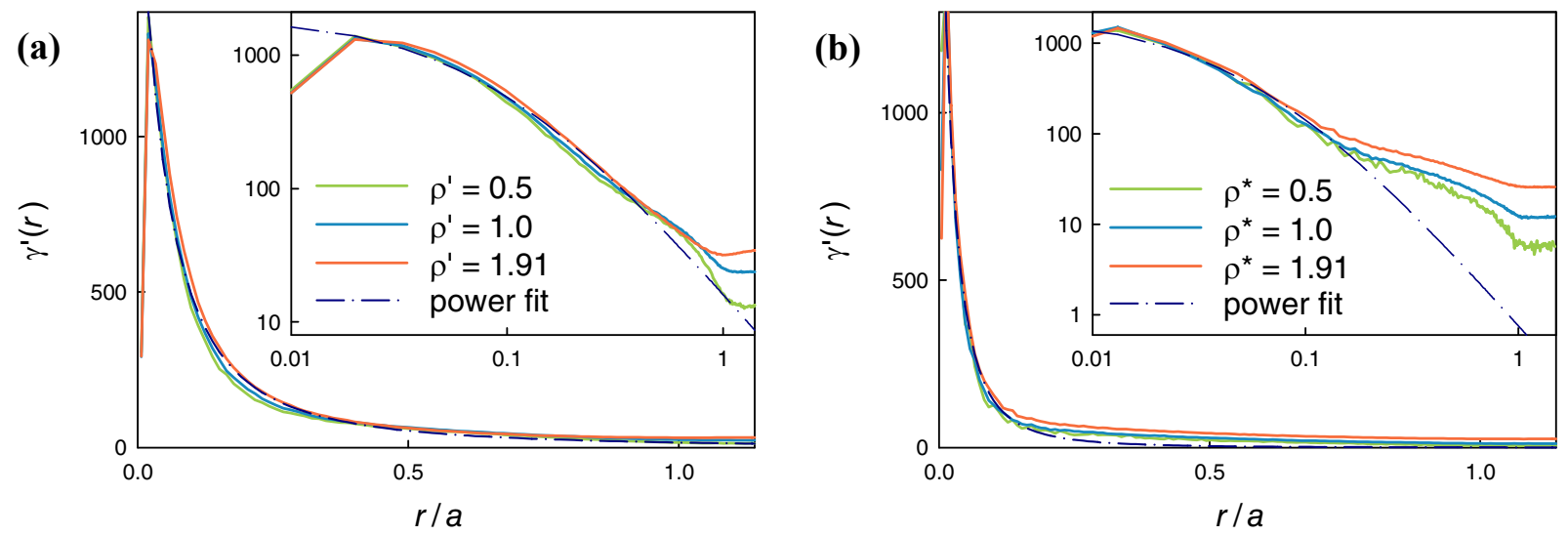

Figure 4. (Colour online) Viscous drag foefficient for several values of BQ density for $a=2 \sigma$ (a) and $a=3 \sigma$ (b). Full lines, simulation results; dashed lines, power fit using Equation B3. Insets show the corresponding log-log plots.

(up to an irrelevant constant) can be directly obtained from Boltzmann inversion, Equation (5). We used this route to calculate the conservative interactions where, in order to improve sampling efficiency in this low-density case, we used a noninteracting 'ensemble' of 1000 BQ pairs.

The conservative potential calculated for all considered BQ sizes is shown in Figure 3. The inset illustrates the damping of repulsion at short range due to correlated motion of the particles at this range leading, for $\mathrm{BQ}$ of smaller sizes, to attraction at extremely short distances. At two larger coarse-graining ratios corresponding to $a=6 \sigma$ and $a=8 \sigma$ the conservative potential decreases monotonously. Solid lines are the results of the fit using Equation (B1). The obtained parameters of conservative potential and force for three smaller quasiparticles are given in Table B1.

In order to obtain the parameters of the dissipative forces, we calculated the distance dependent viscous drag coefficient $\gamma^{\prime}(r)$ using Equation (7). The results, present in Figure 4 for several studied densities for two smaller BQ sizes, $a=2 \sigma$ (a) and $a=3 \sigma$ (b), almost collapsed to a single line in the former case, where the density dependence occurs only at separations greater than the BQ size. For larger coarse-graining ratio the density-dependent hydrodynamic effects are however visible in the BQ overlap region, $a^{\prime}<1$. The parameters of the fits for three smaller BQ cases are given in Table B1.

Finally, the velocity autocorrelation functions (VACF) of BQ particles, $C(t)$, provide a link to 

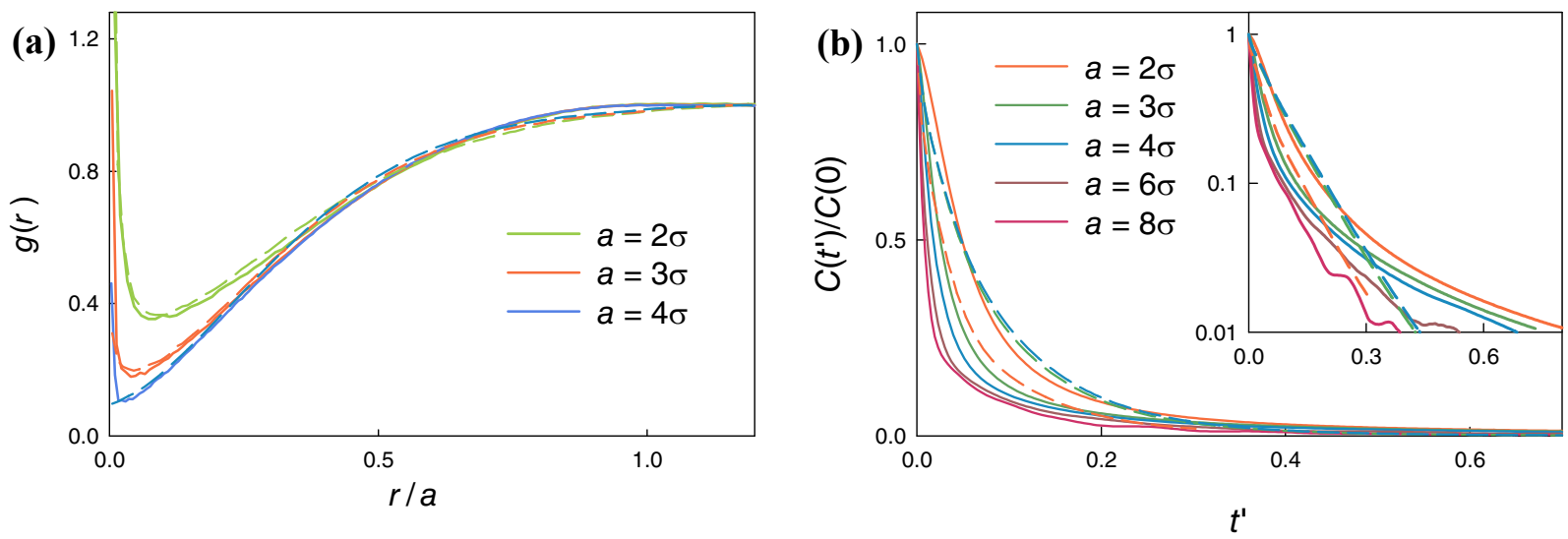

Figure 5. (Colour online) (a) RDFs calculated for the matching BQ (full lines) and DPD (dashed lines) systems for three coarde-graining ratios defined by $a$. (b) Velocity autocorrelation function of DPD particles, averaged over $C_{x x}, C_{y y}$, and $C_{z z}$, for $N=36$ and $a=6 \sigma$. The inset is the $\log -\log$ plot of the same function to illustrate the long-range behaviour. The grey dashed line is the best linear fit for these coordinates at large times.

transport properties of the coarse-grained subsystem. From the energy equipartition it follows that

$$
C(0)=k_{\mathrm{B}} T_{\mathrm{B}}^{*} / \bar{M}_{a}^{*}
$$

where $T_{\mathrm{B}}^{*}$ is the temperature of BQ subsystem (which can deviate from the temperature $T^{*}\left(T^{\prime}=1\right.$ in DPD units) of the atomic host) and $\bar{M}_{a}^{*}$ is the mean mass of a BQ particle. It is related to the coarse-grain ratio, i.e., the mean number of atoms in a BQ, via $\bar{M}_{a}^{*}=N_{m}\left(\rho^{\prime}\right)\left(\right.$ recall that $\left.m^{*}=1\right)$. From (10) we estimate the temperature of the BQ subsystem $T_{\mathrm{B}}^{*}$ and the values of $T_{\mathrm{B}}^{\prime} \equiv T_{\mathrm{B}}^{*} / T^{*}$ for $\rho^{\prime}=1$ are given in Table 1. It occurs that the deviation from 1 at lower values of $a$ is due to the presence of BQs with zero atoms. When for $a^{\prime}=2$ these cases were excluded from the average, temperature dropped to 1.01 .

\subsection{Coarse-grained level}

In order to test and validate the method we performed a series of DPD simulations using the software package DL_MESO [51], which was modified to include the new potential forms for conservative and dissipative forces and to calculate on-the-fly radial distribution and velocity autocorrelation functions. In principle, any molecular dynamics integration scheme can be adapted to integrate the DPD SDE [52]. In practice, however, the velocity Verlet (VV) scheme [34] is often the standard choice. The presence of velocity-dependent Langevin forces require modification of the VV scheme [53], and in our calculations we used DPD-VV scheme as implemented in the DL_MESO package.

Using analytic expressions for the conservative and dissipative forces defined in the appendix by (B2) and (B3), correspondingly, and parameters given in Table B, we performed a series of validating DPD calculations. We used a system containing 1728 DPD beads in a cubic box with the side length of $L=12 a$, which corresponds to a reduced density $\rho^{\prime}=1.0$. To check the accuracy of the conservative forces, we calculated the radial distribution functions of DPD beads and compared them with the reference data obtained for the Brownian quasiparticles (Figure 5a). The differences between the DPD and reference $g(r)$ are comparable with the inaccuracies of the fit, and are smaller than the variation in RDFs due to density changes. This confirms our assumption about the transferability of manybody effects.

Comparison of the DPD velocity autocorrelation functions with BQ for all coarse-graining values 
shown in Figure 5b illustrate the differences in these approaches and highlight the problems of the DPD model. All DPD results are missing the characteristic features of atomistic VACF, which are present in BQ VACF: zero slope at the origin and the hydrodynamic long-range tail. Fitting BQ VACF to an inverse power gave the exponent between 2 and 3 (the last column in Table B1) whereas for DPD the VACF decays exponentially. However, the values of VACF for BQ and DPD are of the same order of magnitude and with a better fit the agreement could be improved.

\section{Conclusions}

We have presented a new approach towards fundamental derivation of model parameters for coarsegraining the nonbonded degrees of freedom within the dissipative particle dynamics framework. Translation of atomistic dynamics onto coarse-grained dissipative dynamics is a complex process and in our approach we used two stages which reflect its different strands and which require separate assumptions. In the first stage, we introduced a Brownian quasiparticle as an open Lagrangian system that could be of any shape. For convenience we considered spherical quasiparticles only but the aspherical case is required to describe the rotational diffusion. The atomic flux in and out of the quasiparticle defines the dissipative and stochastic forces acting on it and the condition that any atom at any time could be a part of at maximum one quasiparticle defines the conservative forces. The atomistic host system drives the dynamics of quasiparticles through the interaction of atoms constituting quasiparticles with the 'environment', i.e., the rest of the atomistic system. At this stage the mapping is straightforward and the motion of quasiparticles gives a faithful description of the fluid motion at the coarse-grained level defined by the dimensions of quasiparticles.

The second stage involves recasting the Brownian quasiparticle dynamics in the form of DPD. This includes several approximations required by the DPD ansatz, including pairwise approximation for all forces, Markovian approximation for Langevin forces [4] and truncation of viscous drag forces at the DPD particle size.

As shown in this work, the proposed method provides a working route to derive the DPD parameters from the underlying atomistic system giving a quantitative agreement of the structure and qualitative description of the dynamics. The proposed concept of Brownian quasiparticle is found to be constructive in revealing the links between the host and coarse-grained description. Focusing on describing the method rather than exploring all its capabilities, we omitted several important quations that need to be investigated further. Thus, an interesting question is whether, in the dependence of coarse-grained dynamics on the bead size, there are any magic numbers. This would be the case if the BQ describe the collective variables in fluid dynamics.

\section{Acknowledgements}

All calculations were performed on Hartree Centre's computers. VPS acknowledges useful discussions with Patrick Warren and Dominic Tildesley.

\section{Funding}

This work was supported in part by funding from the CCP5 Programme. 


\section{References}

[1] Berne BJ, Pecora R. Dynamic light scattering: with applications to chemistry, biology, and physics. New York: Wiley; 1976.

[2] Kamerlin SC, Vicatos S, Dryga A, et al. Coarse-grained (multiscale) simulations in studies of biophysical and chemical systems. Annu Rev Phys Chem. 2011;62:41-64.

[3] Allen MP, Tildesley DJ. Computer simulation of liquids. Oxford: Oxford University Press; 2017.

[4] Zwanzig R. Nonequilibrium statistical mechanics. Oxford: Oxford University Press; 2001.

[5] Zwanzig R. Ensemble method in the theory of irreversibility. J Chem Phys. 1960;33:1338-1341.

[6] Mori H. Transport, collective motion, and Brownian motion. Prog Theor Phys. 1965;33:423-455.

[7] Hijón C, Español P, Vanden-Eijnden E, et al. Mori-Zwanzig formalism as a practical computational tool. Faraday Discuss. 2010;144:301-322.

[8] Cubero D, Yaliraki SN. Formal derivation of dissipative particle dynamics from first principles. Phys Rev E. 2005;72:032101.

[9] Voth GA, editor. Coarse-graining of condensed phase and biomolecular systems. Boca Raton, FL: CRC press; 2008.

[10] Kondov I, Sutmann G, editors. Multiscale modelling methods for applications in materials science. Vol. 19 of IAS Series. Jülich, Forschungszentrum Jülich; 2013.

[11] Akkermans RLC, Briels WJ. Coarse-grained dynamics of one chain in a polymer melt. J Chem Phys. 2000;113:6409-6422.

[12] Akkermans RLC, Briels WJ. A structure-based coarse-grained model for polymer melts. J Chem Phys. 2001;114:1020-1031.

[13] Akkermans RLC, Briels WJ. Coarse-grained interactions in polymer melts: A variational approach. J Chem Phys. 2001;115:6210-6219.

[14] Briels WJ, Akkermans RLC. Representation of coarse-grained potentials for polymer simulations. Mol Simul. 2002;28:145-152.

[15] Allen EC, Rutledge GC. A novel algorithm for creating coarse-grained, density dependent implicit solvent models. J Chem Phys. 2008;128:154115.

[16] Karplus M. Development of multiscale models for complex chemical systems: From H+H2 to biomolecules (Nobel lecture). Angewandte Chemie International Edition. 2014;53:9992-10005.

[17] Levitt M. Birth and future of multiscale modeling for macromolecular systems (Nobel lecture). Ang Chemie Intern Ed. 2014;53:10006-10018.

[18] Warshel A. Multiscale modeling of biological functions: From enzymes to molecular machines (Nobel lecture). Ang Chemie International Edition. 2014;53:10020-10031.

[19] Marrink SJ, Risselada HJ, Yefimov S, et al. The MARTINI force field: coarse grained model for biomol simuls. J Phys Chem B. 2007;111:7812-7824.

[20] Liwo A, Czaplewski C, Pillardy J, et al. Cumulant-based expressions for the multibody terms for the correlation between local and electrostatic interactions in the united-residue force field. J Chem Phys. 2001;115:2323-2347.

[21] Liwo A, Baranowski M, Czaplewski C, et al. A unified coarse-grained model of biological macromolecules based on mean-field multipole-multipole interactions. J Mol Modeling. 2014;20:2306.

[22] Darré L, Machado MR, Brandner AF, et al. SIRAH: A structurally unbiased coarse-grained force field for proteins with aqueous solvation and long-range electrostatics. J Chem Theory Comput. 2015; 11:723-739.

[23] Ercolessi F, Adams JB. Interatomic potentials from 1st-principles calculations - the force-matching method. Europhys Lett. 1994;26:583-588.

[24] McGreevy RL, Pusztai L. Reverse Monte Carlo simulation: A new technique for the determination of disordered structures. Mol Simul. 1988;1:359-367.

[25] McGreevy RL. Reverse Monte Carlo modelling. J Phys Condens Matter. 2001;13:R877-R913.

[26] Shell MS. The relative entropy is fundamental to multiscale and inverse thermodynamic problems. J Chem Phys. 2008;129:144108.

[27] Lopez CF, Nielsen SO, Moore PB, et al. Self-assembly of a phospholipid Langmuir monolayer using coarse-grained molecular dynamics simulations. J Phys Condens Matter. 2002;14:9431.

[28] Qian HJ, Liew CC, Müller-Plathe F. Effective control of the transport coefficients of a coarse-grained liquid and polymer models using the dissipative particle dynamics and Lowe-Andersen equations of 
motion. Phys Chem Chem Phys. 2009;11:1962-1969.

[29] Fu CC, Kulkarni PM, Shell MS, et al. A test of systematic coarse-graining of molecular dynamics simulations: Transport properties. J Chem Phys. 2013;139:094107.

[30] Dünweg B. Molecular dynamics algorithms and hydrodynamic screening. J Chem Phys. 1993;99:69776982.

[31] Liu MB, Liu GR. Particle methods for multi-scale and multi-physics. World Scientific; 2016.

[32] Hoogerbrugge PJ, Koelman JMVA. Simulating microscopic hydrodynamic phenomena with dissipative particle dynamics. Europhys Lett. 1992;19:155-160.

[33] Español P, Warren P. Statistical mechanics of dissipative particle dynamics. Europhys Lett. 1995; 30:191-196.

[34] Groot RD, Warren PB. Dissipative particle dynamics: Bridging the gap between atomistic and mesoscopic simulation. J Chem Phys. 1997;107:4423-4435.

[35] Español P. Hydrodynamics from dissipative particle dynamics. Phys Rev E. 1995;52:1734-1742.

[36] Weeks JD, Chandler D, Andersen HC. Role of repulsive forces in determining the equilibrium structure of simple liquids. J Chem Phys. 1971;54:5237-5247.

[37] Español P, Warren PB. Perspective: Dissipative particle dynamics. J Chem Phys. 2017;146:150901.

[38] Eriksson A, Jacobi MN, Nystrom J, et al. Using force covariance to derive effective stochastic interactions in dissipative particle dynamics. Phys Rev E. 2008;77:016707.

[39] Eriksson A, Jacobi MN, Nystrom J, et al. On the microscopic foundation of dissipative particle dynamics. Europhys Lett. 2009;86:44001.

[40] Eriksson A, Jacobi MN, Nystrom J, et al. A method for estimating the interactions in dissipative particle dynamics from particle trajectories. J Phys Condens Matter. 2009;21:095401.

[41] Español P. Dissipative particle dynamics. In: Grotendorst J, Sutmann G, Gompper G, et al., editors. Hierarchical methods for dynamics in complex molecular systems. Vol. 10 of IAS series; Forschungszentrum Jülich GmbH; 2012. p. 445-468; Available from: http://www.fz-juelich.de/ias/jsc/EN/ Expertise/Workshops/Conferences/WSHD-2012/Publications/Publications .html.

[42] Evans GT. Dissipative particle dynamics: Transport coefficients. J Chem Phys. 1999;110:1338-1342.

[43] Bock H, Gubbins KE, Klapp SHL. Coarse graining of nonbonded degrees of freedom. Phys Rev Lett. 2007;98:267801.

[44] Marsh CA, Backx G, Ernst MH. Static and dynamic properties of dissipative particle dynamics. Phys Rev E. 1997;56:1676-1691.

[45] Zwanzig R. Elementary excitations in classical liquids. Phys Rev. 1967;156:190-195.

[46] Koelman JMVA, Hoogerbrugge PJ. Dynamic simulations of hard-sphere suspensions under steady shear. Europhys Lett. 1993;21:363-368.

[47] Tabor D. Gases, liquids and solids: And other states of matter. Cambridge: Cambridge University Press; 1991.

[48] Henderson RL. A uniqueness theorem for fluid pair correlation functions. Phys Lett A. 1974;49:197-198.

[49] Todorov IT, Smith W, Trachenko K, et al. DL_POLY_3: new dimensions in molecular dynamics simulations via massive parallelism. J Mater Chem. 2006;16:1911-1918.

[50] Ivlev BI. Hydrodynamic fluctuation forces. J Phys: Cond Matt. 2002;14:4829-4842.

[51] Seaton MA, Anderson RL, Metz S, et al. DL_MESO: highly scalable mesoscale simulations. Mol Simul. 2013;39:796-821.

[52] Goga N, Rzepiela AJ, de Vries AH, et al. Efficient algorithms for langevin and dpd dynamics. J Chem Theory Comput. 2012;8:3637-3649.

[53] Vattulainen I, Karttunen M, Besold G, et al. Integration schemes for dissipative particle dynamics simulations: From softly interacting systems towards hybrid models. J Chem Phys. 2002;116:39673979.

\section{Appendix A. A note about the systems of units in DPD modelling}

We used two systems of units defined by the relevant subset of base units that includes length (dimension symbol L), mass (dimension symbol M), and time (dimension symbol $\mathrm{T}$ ). On the atomistic scale it is the Lennard-Jones (LJ) system of units, denoted by an asterisk. They are 
Table B1. Parameters of the fit functions for conservative and dissipative forces.

\begin{tabular}{cccccccc}
\hline$a$ & $A$ & $r_{0}$ & $c$ & $d_{0}$ & $\gamma(0)$ & $r_{\mathrm{d}}$ & $\beta$ \\
\hline $2 \sigma$ & 2.354 & 0.286 & 0.0730 & 0.0210 & 1940 & 0.0932 & 2.00 \\
$3 \sigma$ & 2.870 & 0.247 & 0.0601 & 0.0407 & 5469 & 0.0100 & 1.50 \\
$4 \sigma$ & 2.985 & 0.225 & 0.0361 & 0.0625 & 14773 & 0.0326 & 2.35 \\
\hline
\end{tabular}

sometimes called dimensionless or reduced units. In terms of LJ paricle size, $\sigma$, potential well depth, $\epsilon$, and unified atomic mass unit, $u$, relevant quantities are length $L^{*} \rightarrow L / \sigma$, energy $E^{*} \rightarrow E / \epsilon$, force $F^{*} \rightarrow F \sigma / \epsilon$, viscous drag $\gamma^{*} \rightarrow \gamma \sigma / \sqrt{\epsilon m}$, and diffusivity $D^{*} \rightarrow D \sqrt{m / \epsilon} / \sigma$. On the coarsegrained level, we used a DPD system of units, where a DPD particle size, $s$, defines the length scale and kinetic energy, $k T$, specifies the energy scale.

\section{Appendix B. Details of the fit}

For the Brownin quasiparticles of diameter $s$ we fit the conservative potential to a truncated exponential

$$
U(r)=A \exp \left(-r / r_{0}\right)-c /\left(r+d_{0}\right), \quad(r \leq s)
$$

with the parameters of the fit given in Table B1. This gives rise to the conservative force (4a) with

$$
\alpha(r)=A \exp \left(-r / r_{0}\right) / r_{0}-c /\left(r+d_{0}\right)^{2} .
$$

For the dissipative force (4b), we fit the viscous drag to an inverse power

$$
\gamma(r)=\gamma(0) /\left(1+r / r_{\mathrm{d}}\right)^{\beta}
$$

